\title{
APPROXIMATION THEORY IN THE SPACE OF SECTIONS OF A VECTOR BUNDLE
}

BY

\author{
DAVID HANDEL
}

\begin{abstract}
Let $p: E \rightarrow B$ be a real $m$-plane bundle and $S$ an $n$-dimensional subspace of the space of sections $\Gamma(E)$ of $E$. $S$ is said to be $k$-regular if whenever $x_{1}, \ldots, x_{k}$ are distinct points of $B$ and $v_{i} \in p^{-1}\left(x_{i}\right), 1<i<k$, there exists a $\sigma \in S$ such that $\sigma\left(x_{i}\right)=v_{i}$ for $1<i<k$. It is proved that if $E$ has a Riemannian metric and $B$ is compact Hausdorff with at least $k+1$ points, then $S$ is $k$-regular if and only if for each $\varphi \in \Gamma(E)$, the set of best approximations to $\varphi$ by elements of $S$ has dimension at most $n-k \mathrm{~mm}$. This extends a classical theorem of Haar, Kolmogorov, and Rubinstein (the case of the product line bundle). Complex and quaternionic analogues of the above are obtained simultaneously. Existence and nonexistence of $\boldsymbol{k}$-regular subspaces of a given dimension are obtained in special cases via cohomological methods involving configuration spaces. For example, if $E$ is the product real $(2 m-1)$-plane bundle over a 2-dimensional disk, then $\Gamma(E)$ contains a $k$-regular subspace of dimension $2 \mathrm{~km}-1$, but not one of dimension $2 k m-1-\alpha(k)$, where $\alpha(k)$ denotes the number of ones in the dyadic expansion of $k$.
\end{abstract}

1. Introduction. Let $K$ denote either the real numbers, the complex numbers, or the quaternions and let $p: E \rightarrow B$ be a $K m$-plane bundle. Let $\Gamma(E)$ denote the space of continuous sections of $E$, and let $S$ be a finite-dimensional (over $K$ ) subspace of $\Gamma(E)$.

DefinITION 1.1. $S$ is $k$-regular ( $k$ a positive integer) if whenever $x_{1}, \ldots, x_{q}$ are distinct points of $B, q<k$, and $v_{i} \in p^{-1}\left(x_{i}\right)$ are arbitrarily chosen for $1<i<q$, then there exists a $\sigma \in S$ such that $\sigma\left(x_{i}\right)=v_{i}$ for $1<i<q$.

If $B$ contains at least $k$ points $x_{1}, \ldots, x_{k}$ and $S$ is $k$-regular, then the map $S \rightarrow p^{-1}\left(x_{1}\right) \oplus \cdots \oplus p^{-1}\left(x_{k}\right)$ given by $\sigma \mapsto\left(\sigma\left(x_{1}\right), \ldots, \sigma\left(x_{k}\right)\right)$ is $K$-linear and onto, and so $\operatorname{dim}_{K} S>\mathrm{km}$.

The concept of $k$-regularity will be seen to be of significance in the theory of approximation of arbitrary sections of $E$ by elements of $S$. Suppose $B$ is compact Hausdorff and that $E$ has a Riemannian, Hermitian or symplectic metric (depending on whether $K=R, C$ or $H$ ). Then $\Gamma(E)$ is a Banach space over $K$ with norm given by $\|\varphi\|=\sup \{\|\varphi(x)\| \mid x \in B\}$. Given any $\varphi \in$

Received by the editors June 6, 1978 and, in revised form, February 6, 1979.

AMS (MOS) subject classifications (1970). Primary 55F25, 41A50, 55F40.

Key words and phrases. Vector bundles, best approximation of sections, $k$-regular subspaces of sections, configuration spaces, characteristic classes. 
$\Gamma(E)$, define $d(\varphi, S)=\inf \{\|\varphi-\sigma\| \mid \sigma \in S\}$ and let $\mathscr{B}_{S}(\varphi)=\{\sigma \in S \mid \| \varphi-$ $\sigma \|=d(\varphi, S)\}=$ the set of best approximations to $\varphi$ by elements of $S$. By elementary approximation theory (see, e.g. [1, Chapters I and II]), $\mathscr{B}_{S}(\varphi)$ is a nonempty convex subset of $S$ for each $\varphi \in \Gamma(E)$. In $\$ 2$, the following will be proved:

THEOREM 1.2. Suppose $B$ is compact Hausdorff and contains at least $k+1$ points. Let p: $E \rightarrow B$ be a $K$ m-plane bundle with a metric, and suppose $S$ is an $n$-dimensional (over $K$ ) subspace of $\Gamma(E)$. Then $S$ is $k$-regular if and only if for each $\varphi \in \Gamma(E), \operatorname{dim}_{K} \mathscr{B}_{S}(\varphi)<n-k m$. (The latter condition means that any $n-k m+2$ members of $\mathscr{B}_{S}(\varphi)$ are affinely dependent over $K$.)

In particular, under the above hypotheses, a $k$-regular subspace $S$ will have dimension $\mathrm{km}$ if and only if each $\varphi \in \Gamma(E)$ has a unique best approximation by elements of $S$.

In case $E$ is the product $K$ line bundle over $B, \Gamma(E)$ becomes $C(B, K)$, the space of $K$-valued continuous functions on $B$ with the supremum norm, and Theorem 1.2 specializes to a classical theorem of Haar, Kolmogorov, and Rubinstein (see [10, pp. 237-242]). In this case, $k$-regularity can be reformulated as follows: If $\sigma_{1}, \ldots, \sigma_{n}$ is a $K$-basis for $S \subset C(B, K), S$ is $k$-regular if and only if whenever $x_{1}, \ldots, x_{q}, q<k$, are distinct points in $B$, the vectors $\left(\sigma_{1}\left(x_{i}\right), \ldots, \sigma_{n}\left(x_{i}\right)\right) \in K^{n}, 1 \leqslant i<q$, are linearly independent over $K$.

The proof of Theorem 1.2 is elementary, entailing appropriate extensions of arguments from classical approximation theory (cf. [1, pp. 67-72]), and should be accessible to both topologists and analysts.

The remainder of the paper, which is directed primarily toward algebraic topologists, is concerned with the problem: Given a $K m$-plane bundle $p$ : $E \rightarrow B$, and positive integers $k, n$, does $\Gamma(E)$ contain an $n$-dimensional (over $K$ ) $k$-regular subspace? Some examples with noncompact base spaces are included, as these have approximation-theoretic implications for bundles over compact spaces which are contained in, or contain, these base spaces. In [3], [6], [7] and [8] cohomological methods involving configuration spaces were used to obtain negative results in the real product line bundle case. In \$3, these methods are extended to the general case. In \$4, a number of elementary constructions are given for the manufacture of $k$-regular subspaces, extending some results of [7] and [8]. In $\$ 5$, results of $\$ \S 3$ and 4 are applied to obtain some existence and nonexistence results for $k$-regular subspaces, some of which are best possible. For example, if $E$ is the product real $(2 m-1)$ plane bundle over $R^{2}$, then $\Gamma(E)$ contains a $k$-regular subspace of dimension $2 k m-1$, but not one of dimension $2 k m-1-\alpha(k)$, where $\alpha(k)$ denotes the number of ones in the dyadic expansion of $k$ (a best possible result when $k$ is a power of 2). If $L_{n}$ denotes the canonical real line bundle over real projective 
$n$-space, then $\Gamma\left(L_{n}\right)$ contains a 2-regular subspace of dimension $n+1$, but not one of dimension $n$. $\Gamma\left(L_{n} \oplus L_{n}\right)$ contains a 2-regular subspace of dimension $2 n+2$, but not one of dimension $2 n+1$. If $E$ is the product real 2-plane bundle over a disjoint union of $k$ copies of $S^{2}$, then $\Gamma(E)$ contains a $2 k$-regular subspace of dimension $6 k$, but not one of dimension $6 k-1$.

Aside from any intrinsic interest the results of this paper may hold, the main appeal of this work to algebraic topologists would be the role played by configuration spaces. The latter have figured prominently in a number of other places, e.g. [9], and there is the potential for interaction between the present paper and these other works. In particular it would be interesting to pursue the possible connections of Theorem 1.2 and Proposition 3.1 applied to the tangent bundle of a Riemannian manifold with the work of [9].

The author thanks the referee for a number of helpful suggestions.

2. Best approximation of sections. Throughout this section, $p: E \rightarrow B$ will be a $K m$-plane bundle with a metric appropriate to $K$, and $B$ will be compact Hausdorff.

LEMMA 2.1. Let $S \subset \Gamma(E)$ be $k$-regular, and suppose $B$ contains at least $k+1$ points. Then for each $\varphi \in \Gamma(E)$ and $\sigma \in \mathscr{B}_{S}(\varphi)$, there exist at least $k+1$ points $x$ in $B$ for which $\|\varphi(x)-\sigma(x)\|=d(\varphi, S)$.

Proof. Say $x_{1}, \ldots, x_{r}, r \leqslant k$, are all the points in $B$ for which $\| \varphi(x)-$ $\sigma(x) \|=d(\varphi, S)$. By the $k$-regularity of $S$, there exists $\tau \in S$ such that $\tau\left(x_{i}\right)=\varphi\left(x_{i}\right)-\sigma\left(x_{i}\right)$ for $1 \leqslant i \leqslant r$. Since $\|\varphi(x)-\sigma(x)\|<d(\varphi, S)$ for at least one $x \in B$, we have $d(\varphi, S)>0$.

Write $\rho(x)=\varphi(x)-\sigma(x)$. Since $\rho\left(x_{i}\right)=\tau\left(x_{i}\right)$ for $1<i \leqslant r$, there exists an open subset $U$ of $B$ containing $x_{1}, \ldots, x_{r}$ such that $\|\rho(x)-\tau(x)\|<d(\varphi, S)$ for all $x \in U$. Since $B$ is compact, there exists a real $M>0$ such that $\|\tau(x)\|<M$ for all $x \in B$. Since $B-U$ is compact, $\|\rho(x)\|$ assumes a maximum value $L$ on $B-U$. Since $U$ contains all the $x_{i}$, we have $L<$ $d(\varphi, S)$. Choose $\varepsilon$ so that

$$
0<\varepsilon<\min \left\{\frac{d(\varphi, S)-L}{M}, 1\right\} .
$$

Let $\sigma^{\prime}=\sigma+\varepsilon \tau$. Then $\sigma^{\prime} \in S$. For $x \in U$,

$$
\begin{aligned}
\left\|\varphi(x)-\sigma^{\prime}(x)\right\| & =\|(1-\varepsilon) \rho(x)+\varepsilon \rho(x)-\varepsilon \tau(x)\| \\
& <(1-\varepsilon)\|\rho(x)\|+\varepsilon\|\rho(x)-\tau(x)\| \\
& <(1-\varepsilon) d(\varphi, S)+\varepsilon d(\varphi, S)=d(\varphi, S) .
\end{aligned}
$$

For $x \in B-\mathrm{U}$,

$$
\begin{aligned}
\left\|\varphi(x)-\sigma^{\prime}(x)\right\| & =\|\rho(x)-\varepsilon \tau(x)\|<\|\rho(x)\|+\varepsilon\|\tau(x)\| \\
& <L+\varepsilon M<d(\varphi, S) .
\end{aligned}
$$


Thus $\left\|\varphi-\sigma^{\prime}\right\|<d(\varphi, S)$, a contradiction.

LEMMA 2.2. Let $V_{1}, \ldots, V_{k}$ be finite-dimensional inner-product spaces over $K$ and write $V=V_{1} \oplus \cdots \oplus V_{k}$. Let $W$ be a proper linear subspace of $V$. Then there exists $a\left(v_{1}, \ldots, v_{k}\right) \in V$ such that

(i) $\left\|v_{i}\right\|<1$ for $1<i<k$,

(ii) for each $\left(x_{1}, \ldots, x_{k}\right) \in W, \max \left\{\left\|v_{i}-x_{i}\right\| \mid 1<i<k\right\}>1$.

Proof. For $y=\left(y_{1}, \ldots, y_{k}\right), z=\left(z_{1}, \ldots, z_{k}\right) \in V$ write $\rho(y, z)=$ $\max \left\{\left\|y_{i}-z_{i}\right\| \mid 1<i<k\right\}$ and $\rho(y, W)=\inf \{\rho(y, x) \mid x \in W\}$. Then for each $y \in V$, there exists an $x \in W$ such that $\rho(y, W)=\rho(y, x)$.

Let $C=\left\{\left(v_{1}, \ldots, v_{k}\right) \in V \mid\left\|v_{i}\right\|<1\right.$ for $\left.1<i<k\right\}$. Since $W$ is a proper subspace of $V, C \not W$. Choose any $v^{\prime} \in C-W$ and let $\delta=\rho\left(v^{\prime}, W\right)$. Then $\delta>0$. Choose $w \in W$ satisfying $\rho\left(v^{\prime}, w\right)=\delta$ and set $v=(1 / \delta)\left(v^{\prime}-w\right)$. Then $v \in C$, and for each $x \in W$ we have

$$
\rho(v, x)=\rho\left(\frac{1}{\delta}\left(v^{\prime}-w\right), x\right)=\frac{1}{\delta} \rho\left(v^{\prime}-w, \delta x\right)=\frac{1}{\delta} \rho\left(v^{\prime}, w+\delta x\right) .
$$

Since $w+\delta x \in W$ we have $\rho\left(v^{\prime}, w+\delta x\right)>\delta$, and so $\rho(v, x)>1$ for all $x \in W$.

Proof of Theorem 1.2. Suppose $S$ is $k$-regular and $\varphi \in \Gamma(E)$. Let $q>n-$ $k m+1$ and suppose $\sigma_{1}, \ldots, \sigma_{q} \in \mathscr{B}_{S}(\varphi)$. Then $\tau=q^{-1} \sum_{i=1}^{q} \sigma_{i} \in \mathscr{B}_{S}(\varphi)$. By Lemma 2.1 there exist $k$ distinct points (in fact at least $k+1$ distinct points) $x_{1}, \ldots, x_{k} \in B$ such that $\left\|\varphi\left(x_{i}\right)-\tau\left(x_{i}\right)\right\|=d(\varphi, S)$ for $1<i<k$. Thus, for $1<i<k$,

$$
d(\varphi, S)=\left\|\varphi\left(x_{i}\right)-\tau\left(x_{i}\right)\right\|=\left\|\frac{1}{q} \sum_{j=1}^{q}\left\{\varphi\left(x_{i}\right)-\sigma_{j}\left(x_{i}\right)\right\}\right\| .
$$

Since $\left\|\varphi\left(x_{i}\right)-\sigma_{j}\left(x_{i}\right)\right\|<d(\varphi, S)$ for all $i, j$, it follows that $\varphi\left(x_{i}\right)-\sigma_{j}\left(x_{i}\right)=$ $\varphi\left(x_{i}\right)-\sigma_{1}\left(x_{i}\right)$ for $2<j<q, 1<i<k$. Thus $\left(\sigma_{j}-\sigma_{1}\right)\left(x_{i}\right)=0$ for $1<i<k$, $2<j<q$. Let $f: S \rightarrow p^{-1}\left(x_{1}\right) \oplus \cdots \oplus p^{-1}\left(x_{k}\right)$ be given by $f(\sigma)=$ $\left(\sigma\left(x_{1}\right), \ldots, \sigma\left(x_{k}\right)\right)$. Then $f$ is a $K$-homomorphism which, by the $k$-regularity of $S$, is onto. It follows that the dimension of the kernel of $f$ is $n-k m$. Since $\sigma_{j}-\sigma_{1} \in \operatorname{ker} f$ for $2<j<q$, and $q-1>n-k m$, it follows that the $\sigma_{j}-$ $\sigma_{1}, 2<j<q$, are linearly dependent over $K$, and hence $\sigma_{1}, \ldots, \sigma_{q}$ are affinely dependent over $K$. Thus $\operatorname{dim}_{K} \mathscr{S}_{S}(\varphi)<n-k m$.

Now suppose $S$ is not $k$-regular. Then there exist distinct points $x_{1}, \ldots, x_{k}$ $\in B$ such that the $K$-homomorphism $f: S \rightarrow p^{-1}\left(x_{1}\right) \oplus \cdots \oplus p^{-1}\left(x_{k}\right)$ as above is not onto. Thus it follows from Lemma 2.2 that there exist $v_{i} \in$ $p^{-1}\left(x_{i}\right), \quad 1<i<k$, such that $\left\|v_{i}\right\|<1$ for $1<i<k$, and for each $\left(y_{1}, \ldots, y_{k}\right) \in$ image of $f, \max \left\{\left\|v_{i}-y_{i}\right\| \mid 1<i<k\right\} \geqslant 1$. Using the normality of $B$, there exists $\psi \in \Gamma(E)$ such that $\|\psi(x)\|<1$ for all $x \in B$ and $\psi\left(x_{i}\right)=v_{i}$ for $1<i<k$. Since $\operatorname{dim}_{K} \operatorname{ker} f>n-k m$, there exist linearly 
independent $\sigma_{1}, \ldots, \sigma_{r} \in \operatorname{ker} f$ where $r>n-k m+1$. For $1<i<r$, choose $\lambda_{i}>0$ such that $\sup \left\{\left\|\lambda_{i} \sigma_{i}(x)\right\| \mid x \in B\right\}<1 / r$. Define $\varphi \in \Gamma(E)$ by

$$
\varphi(x)=\left(1-\sum_{i=1}^{r}\left\|\lambda_{i} \sigma_{i}(x)\right\|\right) \psi(x) .
$$

We will show that $\operatorname{dim}_{K} \Re_{S}(\varphi)>r$.

Note that $\varphi\left(x_{i}\right)=v_{i}$ for $1 \leqslant i<k$. By the choice of the $v_{i}$, we have, for each $\sigma \in S$,

$$
\max \left\{\left\|v_{i}-\sigma\left(x_{i}\right)\right\| \mid 1<i<k\right\}>1
$$

and so $d(\varphi, S)>1$. The proof will be completed by showing that $\sum_{i=1}^{r} \varepsilon_{i} \lambda_{i} \sigma_{i}$ $\in \mathscr{B}_{S}(\varphi)$ whenever $\left|\varepsilon_{i}\right|<1$ for $1<i<r$.

For $x \in B$ we have

$$
\begin{aligned}
\| \varphi(x)-\sum_{i=1}^{r} & \varepsilon_{i} \lambda_{i} \sigma_{i}(x)\|<\| \varphi(x)\left\|+\sum_{i=1}^{r}\right\| \varepsilon_{i} \lambda_{i} \sigma_{i}(x) \| \\
& =\|\psi(x)\|\left(1-\sum_{i=1}^{r}\left\|\lambda_{i} \sigma_{i}(x)\right\|\right)+\sum_{i=1}^{r}\left\|\varepsilon_{i} \lambda_{i} \sigma_{i}(x)\right\| \\
& <1-\sum_{i=1}^{r}\left\|\lambda_{i} \sigma_{i}(x)\right\|+\sum_{i=1}^{r}\left|\varepsilon_{i}\right|\left\|\lambda_{i} \sigma_{i}(x)\right\| \\
& =1-\sum_{i=1}^{r}\left(1-\left|\varepsilon_{i}\right|\right)\left\|\lambda_{i} \sigma_{i}(x)\right\|<1 .
\end{aligned}
$$

This, together with the fact that $d(\varphi, S)>1$, implies $d(\varphi, S)=1$ and $\sum_{i=1}^{r} \varepsilon_{i} \lambda_{i} \sigma_{i} \in \mathscr{B}_{S}(\varphi)$.

3. A nonexistence criterion for $k$-regular spaces. Let $F(B, k)$ denote the $k$ th configuration space of $B$, i.e. the subspace of the $k$-fold Cartesian product $B^{k}$ consisting of all $k$-tuples of distinct points. Let $p: E \rightarrow B$ be a $K m$-plane bundle and write

$$
\Phi(E, k)=\left\{\left(v_{1}, \ldots, v_{k}\right) \in E^{k} \mid p\left(v_{i}\right) \neq p\left(v_{j}\right) \text { for } i \neq j\right\} .
$$

Then $q: \Phi(E, k) \rightarrow F(B, k)$ given by $q\left(v_{1}, \ldots, v_{k}\right)=\left(p\left(v_{1}\right), \ldots, p\left(v_{k}\right)\right)$ is a $K k m$-plane bundle (in fact, the restriction of $E^{k}$ to $F(B, k)$ ).

The symmetric group $\Sigma_{k}$ acts freely on $\Phi(E, k)$ and $F(B, k)$ by permuting factors. $q$ is equivariant with respect to these actions. If $B$ is Hausdorff, for each subgroup $G$ of $\Sigma_{k}$, the induced map $\Phi(E, k) / G \rightarrow F(B, k) / G$ is a $K$ $k m$-plane bundle.

Proposition 3.1. If $E$ is as above and if $\Gamma(E)$ admits an n-dimensional (over $K) k$-regular subspace $S$, then for each subgroup $G$ of $\Sigma_{k}, \Phi(E, k) / G$ admits a $K(n-k m)$-plane bundle inverse. 
Proof. If $u$ lies in a space on which $G$ acts, write $[u]$ for its orbit under $G$. The composition

$$
(F(B, k) / G) \times K^{n} \stackrel{f}{\rightarrow}(F(B, k) / G) \times S \stackrel{g}{\rightarrow} \Phi(E, k) / G
$$

where $f$ is the identity crossed with a $K$-isomorphism of $K^{n}$ onto $S$, and $g\left(\left[x_{1}, \ldots, x_{k}\right], \sigma\right)=\left[\sigma\left(x_{1}\right), \ldots, \sigma\left(x_{k}\right)\right]$, is a homomorphism of $K$ vector bundles covering the identity map on $F(B, k) / G$, which, by the $k$-regularity of $S$, is surjective.

REMARK 3.2. If $E_{1}$ and $E_{2}$ are vector bundles over $B$, then

$$
\Phi\left(E_{1} \oplus E_{2}, k\right) / G=\Phi\left(E_{1}, k\right) / G \oplus \Phi\left(E_{2}, k\right) / G .
$$

If $E$ is the product line bundle $B \times K$, then $\Phi(E, k) / G$ can be identified with $F(B, k) \times_{G} K^{k}$, where $G$ acts on $K^{k}$ by permuting factors.

Let $\bar{\sigma}_{i}(\Phi(E, k) / G)$ denote the $i$ th dual characteristic class of $\Phi(E, k) / G$ (Stiefel-Whitney, Chern, or symplectic Pontrjagin, depending on whether $K=R, C$ or $H$ ).

COROLlaRy 3.3. If $E$ is as above and if $\Gamma(E)$ admits an n-dimensional (over $K) k$-regular subspace, then for each subgroup $G$ of $\Sigma_{k}, \bar{\sigma}_{i}(\Phi(E, k) / G)=0$ for $i>n-k m$.

The truth of the converse of Proposition 3.1 in general is unknown, though unlikely. However, for $k=1$ we have:

Proposition 3.4. Let $E$ be as above. Then $\Gamma(E)$ admits $a$ 1-regular subspace of dimension $<n$ over $K$ if and only if $E$ admits a $K(n-m)$-plane bundle inverse.

Proof. If a $K(n-m)$-plane inverse of $E$ existed there would exist a $K$-epimorphism of vector bundles $f: B \times K^{n} \rightarrow E$ covering the identity on $B$. Let $e_{1}, \ldots, e_{n}$ be a basis of $K^{n}$ over $K$ and define $\sigma_{i}: B \rightarrow E$ by $\sigma_{i}(x)=$ $f\left(x, e_{i}\right), 1<i<n$. Then the $\sigma_{i}$ span a subspace of $\Gamma(E)$ of dimension at most $n$ over $K$ which, by the surjectivity of $f$, is 1-regular.

Since $\Phi(E, 1)=E$, the converse follows from Proposition 3.1.

\section{Existence criteria for $k$-regular spaces.}

Proposition 4.1. Let $E_{1}$ and $E_{2}$ be $K$ vector bundles over $B$ and suppose $\Gamma\left(E_{i}\right)$ admits a $k$-regular subspace of dimension $n_{i}$ over $K, i=1,2$. Then $\Gamma\left(E_{1} \oplus E_{2}\right)$ admits a $k$-regular subspace of dimension $n_{1}+n_{2}$ over $K$.

Proof. If $S_{i}$ is an $n_{i}$-dimensional $k$-regular subspace of $\Gamma\left(E_{i}\right), i=1,2$, then $S_{1} \oplus S_{2}$ is the desired $k$-regular subspace of $\Gamma\left(E_{1} \oplus E_{2}\right)$.

Analogous results hold for other functions of vector bundles, e.g. tensor products, exterior powers. 
Proposition 4.2. Let $E=E_{1} \oplus E_{2}$ and suppose $\Gamma(E)$ contains a $k$-regular subspace of dimension $n$ over $K$. Then $\Gamma\left(E_{1}\right)$ contains a $k$-regular subspace of dimension $<n$ over $K$.

Proof. Let $\pi: E \rightarrow E_{1}$ be the projection and suppose $S$ is a $k$-regular subspace of $\Gamma(E)$ of dimension $n$ over $K$. Then $\{\pi \sigma \mid \sigma \in S\}$ is the desired $k$-regular subspace of $\Gamma\left(E_{1}\right)$.

REMARK 4.3. Let $S \subset C\left(K^{q}, K\right)$ be the space of polynomial functions over $K$ of degree $\leqslant k-1(K=R$ or $C)$. It can be shown that $S$ is $k$-regular. Thus, it follows from Propositions 4.1 and 4.2 that if $B$ is a subspace of some $R^{q}$ and $E \rightarrow B$ is a $K$ vector bundle ( $K$ may be $H$, by considering the underlying real or complex structures), then $\Gamma(E)$ contains a finite-dimensional $k$-regular subspace. (When $K=H$ it can be checked that the above $S$ is $k$-regular if $k \leqslant 2$ but fails to be $k$-regular for $k \geqslant 3$.)

Proposition 4.4. Let $p: E \rightarrow B$ be a $K$ m-plane bundle, and $S$ an n-dimensional (over $K$ ) $k$-regular subspace of $\Gamma(E), k \geqslant 2$. Then for each $x_{0} \in B$, $\Gamma\left(E \mid B-\left\{x_{0}\right\}\right)$ contains an $(n-m)$-dimensional (over $\left.K\right)(k-1)$-regular subspace.

Proof. Let $T=\left\{\sigma \in S \mid \sigma\left(x_{0}\right)=0\right\}$. 1-regularity of $S$ implies that $0 \rightarrow T \rightarrow$ $S \stackrel{f}{\rightarrow} p^{-1}\left(x_{0}\right) \rightarrow 0$ is exact, where $f(\sigma)=\sigma\left(x_{0}\right)$, and so $\operatorname{dim}_{K} T=n-m$. Take $T^{\prime}=T \mid\left(B-\left\{x_{0}\right\}\right) . T^{\prime}$ is $(k-1)$-regular, for if $x_{1}, \ldots, x_{q}$ are distinct points of $B-\left\{x_{0}\right\}$ with $q<k-1$ and $v_{i} \in p^{-1}\left(x_{i}\right), 1<i<q$, then $x_{0}, \ldots, x_{q}$ are distinct points of $B$ and so, by the $k$-regularity of $S$, there exists $\sigma \in S$ such that $\sigma\left(x_{0}\right)=0, \sigma\left(x_{i}\right)=v_{i}$ for $1 \leqslant i \leqslant q$.

Proposition 4.4 extends $[8,2.2]$ and $[2$, p. 355].

Proposition 4.5. Let $B$ be a metric space and $p: E \rightarrow B$ a $K$ vector bundle over $B$. Let $A$ be closed in $B$ and suppose $\Gamma(E \mid B-A), \Gamma(E \mid A)$ contain $k$-regular subspaces of dimensions $r$ and $s$, respectively, over $K$. Then $\Gamma(E)$ contains a $k$-regular subspace of dimension $\leqslant r+s$.

Proof. Let $S \subset \Gamma(E \mid B-A), T \subset \Gamma(E \mid A)$ be $k$-regular subspaces with bases $\sigma_{1}, \ldots, \sigma_{r}$ and $\tau_{1}, \ldots, \tau_{s}$, respectively, over $K$. We can assume $E$ has a metric. We can suppose $\left\|\sigma_{i}(x)\right\|$ is bounded for $1<i<r$ (for if we let $f(x)=1+\max _{i}\left\|\sigma_{i}(x)\right\|$, then $\sigma_{1} / f, \ldots, \sigma_{r} / f$ also span a $k$-regular subspace). Choose a continuous $\alpha: B \rightarrow[0,1]$ such that $\alpha(A)=0, \alpha(x)>0$ for $x \in B-$ $A$. Define $\tilde{\sigma}_{i}: B \rightarrow E$ by

$$
\tilde{\sigma}_{i}(x)=\left\{\begin{array}{l}
\alpha(x) \sigma_{i}(x), \quad \text { if } x \in B-A, \\
0, \quad \text { if } x \in A .
\end{array}\right.
$$

Then each $\tilde{\sigma}_{i} \in \Gamma(E)$, and the $\tilde{\sigma}_{i} \mid B-A$ span a $k$-regular subspace $\tilde{S}$ of $\Gamma(E \mid B-A)$. 
Using the normality of $B$, each $\tau_{i}$ extends to a section $\tilde{\tau}_{i}: B \rightarrow E$. Take $U$ to be the space spanned by $\tilde{\sigma}_{1}, \ldots, \tilde{\sigma}_{r}, \tilde{\tau}_{1}, \ldots, \tilde{\tau}_{s} . U$ is $k$-regular, for if $x_{1}, \ldots, x_{i} \in A, y_{i+1}, \ldots, y_{q} \in B-A$ are distinct points with $q<k$, and $v_{j} \in p^{-1}\left(x_{j}\right), w_{j} \in p^{-1}\left(y_{j}\right)$, then the $k$-regularity of $T$ implies there exists a $\tau$ in the span of the $\tilde{\tau}_{j}$ such that $\tau\left(x_{j}\right)=v_{j}$ for $1<j<i$. The $k$-regularity of $\tilde{S}$ implies there exists a $\sigma$ in the span of the $\tilde{\sigma}_{j}$ such that $\sigma\left(y_{j}\right)=w_{j}-\tau\left(y_{j}\right)$ for $i+1 \leqslant j \leqslant q$. Then $\sigma+\tau$ has the required properties.

Proposition 4.5 extends [7, 3.1].

5. Examples. If $E$ denotes the product $K m$-plane bundle over $B$, we identify $\Gamma(E)$ with $C\left(B, K^{m}\right)$, the space of continuous functions of $B$ into $K^{m}$.

Proposition 5.1. Let $K=R$ or $C$. Then for each $k \geqslant 1, C\left(K, K^{m}\right)$ contains a km-dimensional (over $K$ ) $k$-regular subspace.

Proof. The space of polynomial functions over $K$ of degree $<k-1$ is a $k$-dimensional (over $K$ ) $k$-regular subspace of $C(K, K)$. The result now follows from Proposition 4.1.

Note that Proposition 5.1 is a best possible result. Proposition 5.1 also holds when $K=H$ and $k<2$, but the case $K=H, k>3$ is unsettled.

THEOREM 5.2. Let $k$ and $m$ be positive integers and let $\alpha(k)$ denote the number of ones in the dyadic expansion of $k$. Then:

(a) $C\left(R^{2}, R^{2 m}\right)$ contains a $k$-regular subspace of real dimension $2 \mathrm{~km}$.

(b) $C\left(R^{2}, R^{2 m-1}\right)$ contains a $k$-regular subspace of real dimension $2 k m-1$, but not one of the real dimension $2 \mathrm{~km}-1-\alpha(k)$.

Proof. By Proposition 5.1, $C\left(R^{2}, R^{2 m}\right)=C\left(C, C^{m}\right)$ contains a $k$-regular subspace of complex dimension $\mathrm{km}$, and hence of real dimension $2 \mathrm{~km}$, proving (a).

By [3, Example 1.2], $C\left(R^{2}, R\right)$ contains a $k$-regular subspace of real dimension $2 k-1$. The positive part of (b) now follows from this, (a) applied to $C\left(R^{2}, R^{2 m-2}\right)$, and Proposition 4.1.

Write $P_{2, k}$ for the real $k$-plane bundle $F\left(R^{2}, k\right) \times_{\Sigma_{k}} R^{k} \rightarrow F\left(R^{2}, k\right) / \Sigma_{k}$. By [4, Theorem 1], $2 P_{2, k}$ is trivial and so $\bar{w}\left((2 m-1) P_{2, k}\right)=w\left(P_{2, k}\right)$. By [3, Theorem 3.1], $w_{k-\alpha(k)}\left(P_{2, k}\right) \neq 0$. The negative part of (b) now follows from Corollary 3.3 and Remark 3.2.

Theorem 5.2 extends results of [3]. Note that Theorem 5.2(a) is best possible, and Theorem 5.2(b) is best possible when $k$ is a power of 2 .

We recall the following result from [11]:

THEOREM 5.3 (WU). Let $M$ be a closed, connected, $n$-dimensional manifold and let $u$ denote the first Stiefel-Whitney class of the double covering $F(M, 2)$ $\rightarrow F(M, 2) / \Sigma_{2}$. Let $i$ be the largest integer such that $\bar{w}_{i}(M) \neq 0$. Then $u^{n+i} \neq$ $0, u^{n+i+1}=0$. 
LEMMA 5.4. Let $X$ be a Hausdorff space and write $P_{X, 2}=F(X, 2) \times_{\Sigma_{2}} R^{2}$. Let $L$ denote the real line bundle associated with the double covering $F(X, 2) \rightarrow$ $F(X, 2) / \Sigma_{2}$. Then the 2-plane bundle $P_{X, 2} \rightarrow F(X, 2) / \Sigma_{2}$ is the Whitney sum of $L$ and $a$ trivial line bundle.

Proof. $L=F(X, 2) \times_{\Sigma_{2}} R$ where the generator of $\Sigma_{2}$ acts on $R$ by multiplication by -1 . The map $\left(F(X, 2) \times_{\Sigma_{2}} R\right) \times R \rightarrow P_{X, 2}$ given by $\left(\left[x_{1}, x_{2}\right.\right.$; $r], s) \mapsto\left[x_{1}, x_{2} ; s+r, s-r\right]$ is well defined, and is the desired bundle isomorphism.

THEOREM 5.5. Let $M$ be a closed, connected, $n$-dimensional manifold. Let $q$ be the largest integer for which $\bar{w}_{q}(M) \neq 0$. Suppose $C\left(M, R^{m}\right)$ contains a 2-regular subspace of real dimension $N$. Then $\left(\begin{array}{c}m+i-1 \\ m-1\end{array}\right)$ must be even for $N-2 m<i$ $<n+q$.

Proof. By Remark 3.2, $F(M, 2) \times_{\Sigma_{2}}\left(R^{m}\right)^{2}$ can be identified with $m P_{M, 2}$. By Lemma 5.4,

$$
\bar{w}\left(m P_{M, 2}\right)=(1+u)^{-m}=\sum_{i>0}\left(\begin{array}{c}
m+i-1 \\
m-1
\end{array}\right) u^{i}
$$

where $u$ is the first Stiefel-Whitney class of the double cover $F(M, 2) \rightarrow$ $F(M, 2) / \Sigma_{2}$. The result now follows from Corollary 3.3 and Theorem 5.3.

LEMMA 5.6. $C\left(C^{n}, C\right)$ contains a 2-regular subspace of complex dimension $n+1$.

Proof. The space of complex polynomial functions of degree $<1$ is the desired 2-regular subspace.

COROLlaRY 5.7. $C\left(R^{2 n}, R^{2}\right)$ contains a 2-regular subspace of real dimension $2 n+2$.

THEOREM 5.8. Let $M$ be a closed n-dimensional manifold which embeds in $R^{n+k}, n+k$ even. Suppose that either $\bar{w}_{k-2}(M)$ or $\bar{w}_{k-1}(M)$ is nonzero. Then $C\left(M, R^{2}\right)$ contains a 2-regular subspace of dimension $n+k+2$, but not one of dimension $n+k+1$.

Proof. This follows immediately from Corollary 5.7 and Theorem 5.5.

COROllary 5.9. (a) Let $R P^{n}$ denote real projective $n$-space. If $n$ is a power of 2 , then $C\left(R P^{n}, R^{2}\right)$ contains a 2-regular subspace of dimension $2 n+2$, but not one of dimension $2 n+1$.

(b) Let $n$ be odd. Then $C\left(S^{n}, R^{2}\right)$ contains a 2-regular subspace of dimension $n+3$, but not one of dimension $n+2$.

(c) Let $n$ be even. Then $C\left(S^{n}, R^{2}\right)$ contains a 2-regular subspace of dimension $n+4$, but not one of dimension $n+3$. 
THEOREM 5.10. Let $X$ be the disjoint union of $k$ copies of $S^{2}$. Then $C\left(X, R^{2}\right)$ contains a $2 k$-regular subspace of dimension $6 k$, but not one of dimension $6 k-1$.

Proof. Let $D$ be a $k$ point subspace of $X$ consisting of one point from each $S^{2}$. Then $X-D$ is embeddable in $R^{2}$, and so by Theorem 5.2, $C\left(X-D, R^{2}\right)$ contains a $2 k$-regular subspace of dimension $4 k$. Clearly, $C\left(D, R^{2}\right)$ contains a $2 k$-regular subspace of dimension $4 k$. Clearly, $C\left(D, R^{2}\right)$ contains a $2 k$-regular subspace of dimension $2 k$. Thus by Proposition 4.5, $C\left(X, R^{2}\right)$ contains a $2 k$-regular subspace of dimension $<6 k$.

Let $G=\Sigma_{2} \times \cdots \times \Sigma_{2} \subset \Sigma_{2 k}$ where there are $k$ factors $\Sigma_{2}$ and where the generator of the $i$ th factor is the transposition which interchanges $2 i-1$ and $2 i, 1<i<k$. To show that $C\left(X, R^{2}\right)$ does not contain a $2 k$-regular subspace of dimension $<6 k$ it suffices to show, by Corollary 3.3 and Remark 3.2, that $\bar{w}_{2 k}\left(F(X, 2 k) \times_{G}\left(R^{2}\right)^{2 k}\right) \neq 0$. There is an evident map of $4 k$-plane bundles

$$
\begin{aligned}
& E=\left[F\left(S^{2}, 2\right) \times_{\Sigma_{2}}\left(R^{2}\right)^{2}\right] \times \cdots \times\left[F\left(S^{2}, 2\right) \times_{\Sigma_{2}}\left(R^{2}\right)^{2}\right] \\
& \downarrow \\
& F\left(S^{2}, 2\right) / \Sigma_{2} \times \cdots \times F\left(S^{2}, 2\right) / \Sigma_{2} \\
& \rightarrow \quad F(X, 2 k) \times_{G}\left(R^{2}\right)^{2 k} \\
& \downarrow \\
& \rightarrow \quad F(X, 2 k) / G
\end{aligned}
$$

and so it suffices to show that $\bar{w}_{2 k}(E) \neq 0$. If $u$ denotes the first StiefelWhitney class of the double covering $F\left(S^{2}, 2\right) \rightarrow F\left(S^{2}, 2\right) / \Sigma_{2}$, it follows from Lemma 5.4 that

$$
w\left(F\left(S^{2}, 2\right) \times_{\Sigma_{2}}\left(R^{2}\right)^{2}\right)=w\left(F\left(S^{2}, 2\right) \times_{\Sigma_{2}} R^{2}\right)^{2}=(1+u)^{2}=1+u^{2} .
$$

By Theorem 5.3, $u^{2} \neq 0, u^{3}=0$. Thus $\bar{w}\left(F\left(S^{2}, 2\right) \times_{\Sigma_{2}}\left(R^{2}\right)^{2}\right)=1+u^{2}$ and so $\bar{w}(E)=\left(1+u^{2}\right) \times \cdots \times\left(1+u^{2}\right)$ and $\bar{w}_{2 k}(E)=u^{2} \times \cdots \times u^{2} \neq 0$, each containing $k$ factors.

Let $L_{n}$ denote the canonical real line bundle over $R P^{n}$. We regard $R P^{n}$ as the quotient space obtained from $S^{n}$ by identifying $x \sim-x, x \in S^{n}$, and $L_{n}$ as the quotient space obtained from $S^{n} \times R$ by identifying $(x, t) \sim$ $(-x,-t),(x, t) \in S^{n} \times R$. Write $[x]$ and $[x, t]$, respectively, for the points in $R P^{n}$ and $L_{n}$, respectively, determined by $x \in S^{n},(x, t) \in S^{n} \times R$.

THEOREM 5.11. $\Gamma\left(L_{n}\right)$ contains a 2-regular subspace of dimension $n+1$, but not one of dimension $n$.

Proof. If $x=\left(x_{1}, \ldots, x_{n+1}\right) \in S^{n}$, define $\sigma_{i} \in \Gamma\left(L_{n}\right)$ by $\sigma_{i}[x]=\left[x, x_{i}\right]$, $1<i<n+1$. Let $S$ be the subspace spanned by the $\sigma_{i}$. It is easily checked that $S$ is 2 -regular and $n+1$-dimensional over $R$. 
Suppose $\Gamma\left(L_{n}\right)$ contained a 2-regular subspace of dimension $n$. Then by Proposition 4.4, for each $x_{0} \in R P^{n}, \Gamma\left(L_{n} \mid R P^{n}-\left\{x_{0}\right\}\right)$ would contain a 1-regular subspace of dimension $n-1$. It follows that $\Gamma\left(L_{n-1}\right)$ would contain a 1-regular subspace of dimension $n-1$. Thus by Proposition $3.4, L_{n-1}$ would admit an $(n-2)$-plane inverse, which is seen to be false by a Stiefel-Whitney class argument.

THEOREM 5.12. $\Gamma\left(L_{n} \oplus L_{n}\right)$ contains a 2-regular subspace of dimension $2 n+2$, but not one of dimension $2 n+1$.

Proof. The existence part follows from Theorem 5.11 and Proposition 4.1. For the nonexistence part it suffices to show, by Corollary 3.3 and Remark 3.2 , that $\bar{w}_{2 n-2}\left(2 \Phi\left(L_{n}, 2\right) / \Sigma_{2}\right) \neq 0$. Following the notation of [5], let $S Z_{n+1,2}$ denote the subspace of $F\left(R P^{n}, 2\right) / \Sigma_{2}$ consisting of the unordered pairs of points $\{[x],[y]\}$ with $x$ orthogonal to $y$. Let $E$ be the restriction of $\Phi\left(L_{n}, 2\right) / \Sigma_{2}$ to $S Z_{n+1,2}$. It suffices to show that $\bar{w}_{2 n-2}(2 E) \neq 0$. Consider the dihedral group

$$
G=\left\{\left(\begin{array}{cc}
\varepsilon_{1} & 0 \\
0 & \varepsilon_{2}
\end{array}\right),\left(\begin{array}{cc}
0 & \varepsilon_{3} \\
\varepsilon_{4} & 0
\end{array}\right) \mid \varepsilon_{i}= \pm 1\right\}
$$

$G$ acts freely on the Stiefel manifold $V_{n+1,2}$ of orthonormal 2-frames in $R^{n+1}$ as in [5], and $S Z_{n+1,2}$ is precisely the orbit space of this action. $E$ can be identified with $V_{n+1,2} \times{ }_{G} R^{2}$ where $G$ acts on $R^{2}$ as a subgroup of $O(2)$. In the notation of [5], the composition

$$
S Z_{n+1,2} \stackrel{p_{2}}{\rightarrow} B G \stackrel{i_{1}}{\rightarrow} B O(2)
$$

is a classifying map for $E$, where $p_{2}$ classifies the principal $G$-bundle $V_{n+1,2} \rightarrow$ $S Z_{n+1,2}$ and $i_{1}$ is induced by the inclusion $G \subset O(2)$. In the notation of [5], $w(E)=1+y_{1}+y_{2}$. Let $Z_{n+1,2}$ denote the subspace of $F\left(R P^{n}, 2\right)$ consisting of all $([x],[y])$ with $x$ orthogonal to $y$, and let $\pi_{1}: Z_{n+1,2} \rightarrow S Z_{n+1,2}$ denote the projection. $Z_{n+1,2}$ is the projective bundle associated to the tangent bundle of $R P^{n}$. Writing $v$ for the first Stiefel-Whitney class of the canonical line bundle over $Z_{n+1,2}, H^{*}\left(Z_{n+1,2} ; Z_{2}\right)$ is the free $H^{*}\left(R P^{n} ; Z_{2}\right)$-module with basis $1, v$, $v^{2}, \ldots, v^{n-1}$. Write $z$ for the generator of $H^{1}\left(R P^{n} ; Z_{2}\right)$. In [5] it is proved that $\pi_{1}^{*}\left(y_{1}\right)=v, \pi_{1}^{*}\left(y_{2}\right)=z^{2}+z v$. A rather long, but straightforward, computation using $[5,3.1]$ shows that $\pi_{1}^{*} \bar{w}_{2 n-2}(2 E)=z^{n-1} v^{n-1}+$ terms of lower degree in $v$, proving $\bar{w}_{2 n-2}(2 E) \neq 0$.

\section{REFERENCES}

1. N. I. Ahiezer, Theory of approximation, Frederick Ungar, New York, 1956.

2. K. Borsuk, On the k-independent subsets of the Euclidean space and of the Hilbert space, Bull. Acad. Polon. Sci. Cl. III 5 (1957), 351-356.

3. F. R. Cohen and D. Handel, $k$-regular embeddings of the plane, Proc. Amer. Math. Soc. 72 (1978), 201-204. 
4. F. R. Cohen, M. E. Mahowald and R. J. Milgram, The stable decomposition for the double loop space of a sphere, Algebraic and Geometric Topology, Proc. Sympos. Pure Math, vol. 32, part 2, Amer. Math. Soc., Providence, R. I., 1978, pp. 225-228.

5. D. Handel, An embedding theorem for real projective spaces, Topology 7 (1968), 125-130.

6. ___, Obstructions to 3-regular embedings, Houston J. Math. (to appear).

7. appear).

8. D. Handel and J. Segal, On k-regular embeddings of spaces in Euclidean space, Fund. Math. (to appear).

9. D. McDuff, Configuration spaces of pasitioe and negative particles, Topology 14 (1975), 91-107.

10. I. Singer, Best approximation in normed linear spaces by elements of linear subspaces, Springer-Verlag, Berlin and New York, 1970.

11. W-T. Wu, On the realization of complexes in euclidean spaces. II, Acta Math. Sinica 7 (1957), 79-101.

Department of Mathematics, Wayne State Universtry, Detrott, Michoan 48202 